\title{
Pulse Transmission across a Single Optical Ring- Resonator with Negative Group Velocity: Theory and Experiment
}

\author{
H. P. Uranus ${ }^{1,2, *}$, L. Zhuang ${ }^{3}$, C. G. H. Roeloffzen ${ }^{3}$, and H. J. W. M. Hoekstra ${ }^{4}$ \\ ${ }^{1}$ The Graduate Program in Electrical Engineering, University of Pelita Harapan, \\ Wisma Slipi, Jl. Let. Jend. S. Parman Kav. 12, Jakarta 11480, Indonesia. \\ ${ }^{2}$ Department of Electrical Engineering, The Undergraduate Program, University of Pelita Harapan, \\ Lippo Karawaci, Tangerang 15811, Indonesia. \\ ${ }^{3}$ Telecommunication Engineering Group, Dept. of Electrical Engineering, University of Twente, \\ P. O. Box 217, 7500 AE Enschede, The Netherlands \\ ${ }^{4}$ Integrated Optical Microsystems Group, Dept. of Electrical Engineering, University of Twente, \\ P. O. Box 217, 7500 AE Enschede, The Netherlands \\ E-mail: h.p.uranus@alumnus.utwente.nl
}

\begin{abstract}
We study pulse transmission across a single optical ring-resonator circuit theoretically. The theory is supported by experimental measurements. From the study, we discuss intuitively the fulfillment of the causality rules in negative delay phenomenon, which occurs when the circuit possesses a negative group velocity.
\end{abstract}

\section{INTRODUCTION}

Group velocity $\left(v_{\mathrm{g}}\right)$ of lightwave traveling across an optical structure is defined as

$v_{g} \equiv\left\{\partial \beta_{\text {eff }} / \partial \omega\right\}^{-1}$,

where $\beta_{\text {eff }}$ and $\omega$ are the effective propagation constant and angular frequency, respectively; is the velocity of the envelope of a superposition of a group of waves with slightly different frequencies. By substituting $\beta_{\text {eff }}=\omega n_{\text {eff }} / c$ with $n_{\text {eff }}$ and $c$ are the effective index of the structure and the light velocity in vacuum, respectively, into Eq. (1), we arrive at

$v_{\mathrm{g}}=c / n_{\mathrm{g}}$,

where the group index $n_{\mathrm{g}}$ is

$n_{\mathrm{g}}=n_{\text {eff }}+\omega \partial n_{\text {eff }} / \partial \omega$.

Eq. (3) implies that we can control the $v_{\mathrm{g}}$ by the structural dispersion sitting at the second term of the equation. The group delay $\tau_{g}$ is defined as

$\tau_{g} \equiv L / v_{g}$

with $L$ is the length of the optical structure.

If the dispersion is anomalous, the second term of Eq. (3) will be negative. An exotic phenomenon will happen when this term is stronger than the first term, since $v_{\mathrm{g}}$ will be negative. Negative $v_{\mathrm{g}}$ implies that if we send light pulse into the optical structure, the peak of the output pulse will leave the output before the peak of the input pulse entering the structure [1]-[3], i.e. a pulse 'advancement' or negative group delay (a negative $\tau_{\mathrm{g}}$ for a positive $L$ in Eq. (4)). It also implies that inside the medium, there is a backward pulse propagation [4], i.e. a negative $L$ for a positive $\tau_{\mathrm{g}}$ in Eq. (4), where here $L$ represents the propagation distance. Although these effects, which in fact have been observed in experiments [2]-[4], sound counter-intuitive, their consistency with causality principles has been well verified experimentally [5].

In fact, negative $v_{\mathrm{g}}$ phenomena is not a new subject, as it has been theoretically studied since a few decades ago [6] and observed experimentally e.g. in photonic crystals, atomic gases, active optical fibers, and left-handed materials. A single resonator (not necessarily coupled resonators), is an extremely dispersive device in the neighborhood of its resonant frequency. Consequently, the negative $v_{\mathrm{g}}$ phenomenon can take place in a properly designed resonator, e.g. in an integrated-optical ring-resonator circuit as depicted in Fig. 1.

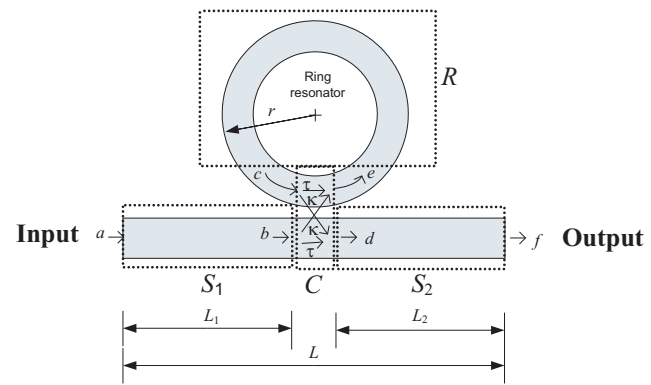

Fig. 1. A two-port integrated-optical ring-resonator circuit under study.

Negative $v_{\mathrm{g}}$ in ring-resonators has been theoretically studied [7], [8]. Recently, we reported a first direct experimental measurement on the pulse temporal behavior passing across an integrated-optical ring-resonator circuit [2]

This work was supported by the Dutch Applied Technology Foundation STW through project TOE.6596 and the Dutch Ministry of Economic Affair through SenterNovem project IS052081. 
with negative and positive $v_{\mathrm{g}}$. In this paper, we present our work on the theory of pulse transmission across the ringresonator circuit with negative and positive $v_{\mathrm{g}}$ [8] and support it by time-domain experimental verification [2] using a real integrated-optical chip. Agreement between both results leads to a better understanding of such phenomenon, including the fulfillment of causality rules.

\section{THE THEORY}

Applying a transfer matrix method onto the model of the ring resonator circuit shown in Fig. 1 (which is assumed to be made of single mode waveguides and owns a lossless coupler) gives

$$
T \equiv \frac{f}{a}=\exp \left(-i \beta_{\text {straight }} L\right) \frac{\tau-\exp (-i \theta)}{1-\tau \exp (-i \theta)}
$$

where $\beta_{\text {straight }}, \tau$, and $\theta$ denote the propagation constant of the straight waveguide, the through amplitude coupling constant of the coupler section, and a complex round trip phase shift around the ring waveguide, respectively. The phase shift can be written as

$$
\theta=\left(\beta_{\text {res }}-i \alpha_{\text {res,lin }}\right) 2 \pi r,
$$

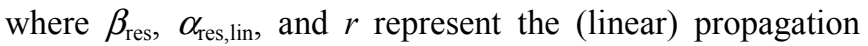
constant, the attenuation constant (in linear scale), and the effective radius of the ring resonator, respectively. By rewriting the transfer function in a complex expression $T=|T| \exp (-i \phi)$, using $\beta_{\text {eff }} \equiv \phi / L$, Eqs. (1) and (2), we arrive at analytical expression of group index

$$
\begin{aligned}
& n_{g}=n_{\text {eff,straight }}+ \\
& \frac{2 \pi r n_{\text {eff,res }}}{L} \frac{\left(1-\tau^{2}\right) \gamma\left[\gamma\left(1+\tau^{2}\right)-\left(1+\gamma^{2}\right) \tau \cos \theta_{\mathrm{R}}\right]}{\left(\tau^{2}-2 \gamma \tau \cos \theta_{\mathrm{R}}+\gamma^{2}\right)\left(\tau^{2} \gamma^{2}-2 \tau \gamma \cos \theta_{\mathrm{R}}+1\right)}
\end{aligned}
$$

where $n_{\text {eff,straight }}, n_{\text {eff,res, }}$, and $\theta_{\mathrm{R}}$ represent the effective index of the straight waveguide, ring waveguide, and the real part of $\theta$, respectively. In Eq. (7), $\gamma$ represents the round trip reduction of amplitude $\gamma=\exp \left(\theta_{\mathrm{I}}\right)$, where $\theta_{\mathrm{I}}$ is the imaginary part of $\theta$.

To study a pulse transmission across the ring-resonator circuit, we express the field entering the input of the circuit as

$$
a(t)=a_{e n v}(t) \exp \left(i \omega_{\mathrm{c}} t\right),
$$

where $a_{\text {env }}(t)$ is the envelope function of the light pulse (i.e. the modulating signal), $t$ is the time relative to a reference time (for which, we took the time of the peak of $a_{\text {env }}(t)$ ), while $\omega_{\mathrm{c}}=2 \pi \mathrm{c} / \lambda_{\mathrm{c}}$ is the carrier angular frequency with $\lambda_{\mathrm{c}}$ is the resonant wavelength of the resonator. This input pulse is sampled and Fourier transformed into

$$
A\left(\lambda_{\mathrm{k}}\right)=\sum_{\mathrm{i}} a\left(t_{\mathrm{i}}\right) \exp \left(-i 2 \pi c t_{\mathrm{i}} / \lambda_{\mathrm{k}}\right) \Delta t
$$

at each discrete wavelength $\lambda_{\mathrm{k}}$. The output signal in frequency domain at each discrete wavelength $\lambda_{\mathrm{k}}$ is simply a multiplication between Eq. (9) and the transfer function Eq. (5) at that wavelength

$$
F\left(\lambda_{\mathrm{k}}\right)=A\left(\lambda_{\mathrm{k}}\right) T\left(\lambda_{\mathrm{k}}\right) \text {. }
$$

Taking inverse Fourier transform onto the frequency domain expression of the output of the circuit gives

$$
f\left(t_{\mathrm{i}}\right)=\sum_{\mathrm{k}} F\left(\lambda_{\mathrm{k}}\right) \exp \left(i 2 \pi c t_{\mathrm{i}} / \lambda_{\mathrm{k}}\right)\left(-c \Delta \lambda / \lambda_{\mathrm{k}}^{2}\right) \text {, }
$$

which allows us to plot the temporal shape of the power of the output pulse $|f(t)|^{2}$. Similarly, we can also plot the temporal shape of the power of the input pulse $|a(t)|^{2}=\left|a_{e n v}(t)\right|^{2}$.

\section{THE EXPERIMENT}

For the experiment, we used a $\mathrm{Si}_{3} \mathrm{~N}_{4}$-based integratedoptical chip which was initially fabricated for optical beamforming application [9]. The chip was realized using the TriPleX waveguide technology [10] by Lionix B.V. [11], The Netherlands. The chip has many race-track ringresonators with thermo-optically controllable couplingconstant (through modulator TO1, see Fig. 2) and resonant wavelength (through modulator TO2), which suits very well to our purpose. We only use one of the ring-resonators. The ring has geometric round trip length $L_{\mathrm{rt}}$ of $11916.4 \mu \mathrm{m}$. Using a mode solver, we compute the mode of the TriPleX waveguide at $\lambda_{0}=1.55 \mu \mathrm{m}$, which results in single q-TE mode with $n_{\text {eff,straight }}=n_{\text {eff,res }}=1.6499$ and $\partial n_{\text {eff,straight }} / \partial \lambda=$ $\partial n_{\text {eff,res }} / \partial \lambda=-0.1029 \mu \mathrm{m}^{-1}$. These values give free spectral range of $13.9 \mathrm{GHz}$, which was well verified by measurement. For further details on the chip, we refer to Ref. [9] and [10].

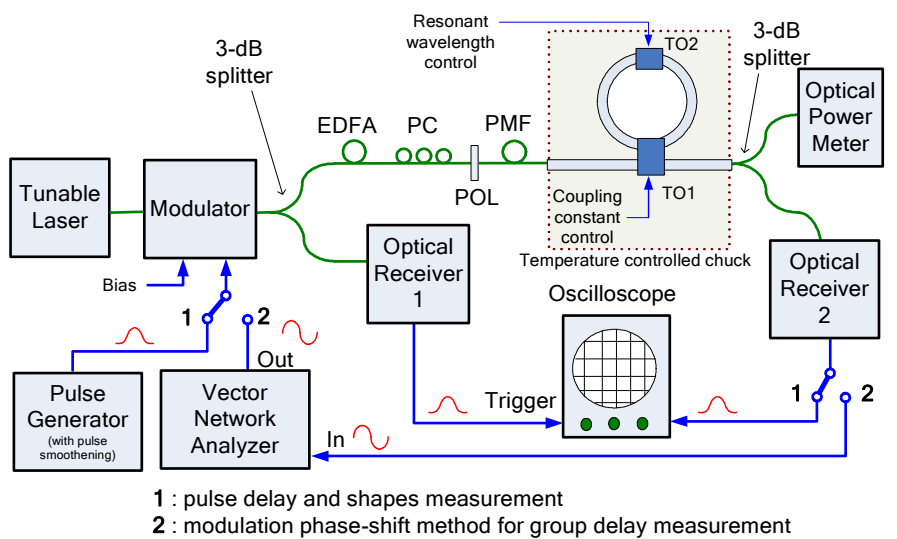

Fig. 2. Schematic picture of the measurement set-up. EDFA = erbium-doped fiber amplifier, $\mathrm{PC}=$ polarization controller, $\mathrm{POL}=$ polarizer, $\mathrm{PMF}=$ polarization maintaining fiber, $\mathrm{TO}=$ thermo-optic modulator

Fig. 2 shows the measurement set-up. Light from a tunable laser (Santec TSL-210) was modulated by an external modulator and sent through a series of fiber optic components to the ring-resonator chip under study. The smooth modulating signal came from a pulse generator (HP 8082A) which owns an internal electronic pulse shaper to control the slope of the pulse's leading and trailing edges. The light was conditioned by the polarization controller, inline polarizer, and polarization maintaining fiber to excite the chip with a TE-polarization. The chip was hold by a thermo-electrically temperature-stabilized chuck for a 
repeatable thermo-optic control over the ring-resonator's parameters. The output of the chip was captured by an optical power meter and the Optical Receiver 2. The output signal's shape and phase were monitored by an oscilloscope triggered by part of the input light captured by Optical Receiver 1. In order to compensate the insertion losses in the set-up and obtain a well-detectable output signal, an erbiumdoped fiber amplifier was inserted into the set-up.

Since a delay or advancement needs reference, we firstly recorded a reference signal, which should be well defined. For this purpose, we initially set the tunable laser into the resonant wavelength of the ring-resonator nearest to $\lambda_{0}$ and then set the coupling constant through TO1 to produce the weakest output signal, which is corresponding to a welldefined critical-coupling condition [8]. Then the TO2 was tuned to operate the ring into the anti-resonant condition, i.e. a condition located at the middle between 2 consecutive resonant wavelengths. Note that, we chose to control TO2 instead of the tunable laser due to a better repeatability of TO2 control. At this anti-resonant condition, the effect of the ring is minimal, and the delay of the circuit is approximately the same as the one contributed by similar circuit without the ring. We denote the pulse captured under this condition as the reference pulse and the associated structure as a reference structure. We then set $\mathrm{TO} 2$ to tune the ring back into resonance. The output pulses at various values of coupling constant tuned by TO1 into under-coupling, criticalcoupling, and over-coupling regimes were recorded as well.

In addition, we also measure the average output power spectrum of the circuit by scanning the tunable laser and reading out the power meter. For this purpose, we switched the set-up to position 2 (see Fig. 2), where a sinusoidal signal of $5 \mathrm{MHz}$ (generated by a vector network analyzer VNA, Agilent N5230A) was employed as the modulating signal. Note that, throughout the experiment, the modulator is DC biased such that the output signal was not distorted. Simultaneously, within each scan step, we also measure the phase difference $(\varphi)$ between the received and sent-out sine signal of the VNA. Using such a modulated phase shift method [12], we can retrieve the relative group delay by

$\tau_{\mathrm{g}, \mathrm{rel}}=\frac{\varphi}{2 \pi f_{\mathrm{m}}}-\tau_{\mathrm{g}, \mathrm{ref}}$

where $f_{\mathrm{m}}$ and $\tau_{\mathrm{g}, \mathrm{ref}}$ are the modulating frequency and the group delay of the reference structure measured earlier, respectively.

\section{RESULTS AND DISCUSSIONS}

Fig. 3 shows the measured output pulses for various settings of TO1 (i.e. various values of the coupling constant) along with the reference pulse. We refer the position of the peak of the reference pulse as $t=0$. Since the reference structure approximately represents a ring-less structure, the pulse positions in Fig. 3 represent the delay contributed by the ring-resonator. It is clear from the figure that when the TO1 was tuned to coupling constant values of $\kappa_{5}, \kappa_{6}$, and $\kappa_{7}$, the peak of the output pulse came out earlier than the peak of the reference pulse. This is an experimental verification of the counter-intuitive phenomenon of negative group delay as pointed out in the introduction of this paper. Our experiment shows that the weakest output signal happened at $\kappa_{4}$, which resulted in a pulse with two peaks. This extreme condition is associated with the critical coupling condition [8].

In these measurement results, the causality relation is not violated at all. Although in the negative $v_{\mathrm{g}}$ regime (denoted by coupling constants $\kappa_{5}$ to $\kappa_{7}$ ), the peak of the output pulse leaves the output before the peak of the reference pulse, it experiences a loss which makes its peak always lays below the rising edge of the reference pulse. This implies that the energy velocity is in fact positive, i.e. no energy can come out of the circuit before input energy going into the circuit. A more intuitive explanation is through the mathematical meaning of an analytic smooth pulse [13] like the Gaussian pulse, which is approximated in the experiments. An analytic smooth function is (infinitely) differentiable, and hence able to be expressed in a Taylor's expansion. Taylor's expansion means that we are able to express the value of the future with values in the vicinity of a point in the past. Hence, in an analytic smooth function, the future is already known from the past. Hence, information velocity is still positive even when the $v_{\mathrm{g}}$ is negative. In that condition, $v_{\mathrm{g}}$ is no more the information velocity as already stated by Brillouin [6]. If the pulse is not fully analytic, the Taylor's expansion should be truncated, leading to a loss of information. We can intuitively see from Fig. 3, that the more the pulse is advanced, the heavier the distortion is. Distortion implies a loss of information. The heaviest distortion happens when the negative $v_{\mathrm{g}}$ is in its extreme point, i.e. at the critical coupling point, where the pulse apparently splits into two sub-pulses.

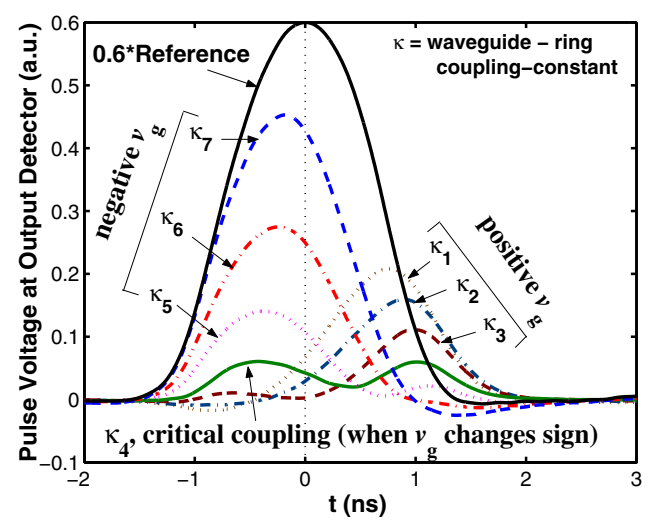

Fig. 3. The measured output pulse shape and delay for various values of coupling constant set by TO1.

The circles in Fig. 4.(a) indicate the measured output power spectrum. The same symbols in Fig. 4.(b) indicate the measured relative group delay for the same coupling constant settings as in Fig. 3. Using the measured data in Fig. 4, we perform a curve fitting with respect to their theoretical expressions. The best fit values for $\kappa_{1}, \kappa_{2}, \kappa_{3}, \kappa_{5}, \kappa_{6}, \kappa_{7}$, and ring attenuation constant $\alpha_{\text {res }}=20 \alpha_{\text {res, } \operatorname{lin}} \log (e)$ are $-0.5984 \mathrm{i}$, $-0.5542 \mathrm{i},-0.5223 \mathrm{i},-0.3581 \mathrm{i},-0.3196 \mathrm{i},-0.2695 \mathrm{i}$, and 0.7842 
$\mathrm{dB} / \mathrm{cm}$, respectively. Since it is difficult for a phase shift method to measure a sharp feature [12] in the neighborhood of critical coupling point, we intentionally exclude measured result for $\kappa_{4}$ from the fitting procedure while other curves are fitted altogether. The $\kappa$ value for the critical coupling point is calculated from the fitted $\alpha_{\text {res }}$ and $L_{\mathrm{rt}}$ which gives $-0.44 \mathrm{i}$. The best fit theoretical curves are plotted in solid lines in Fig. 4.

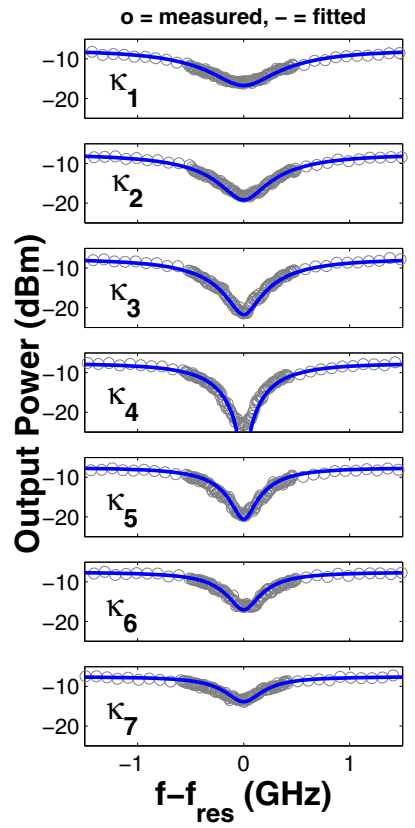

(a)

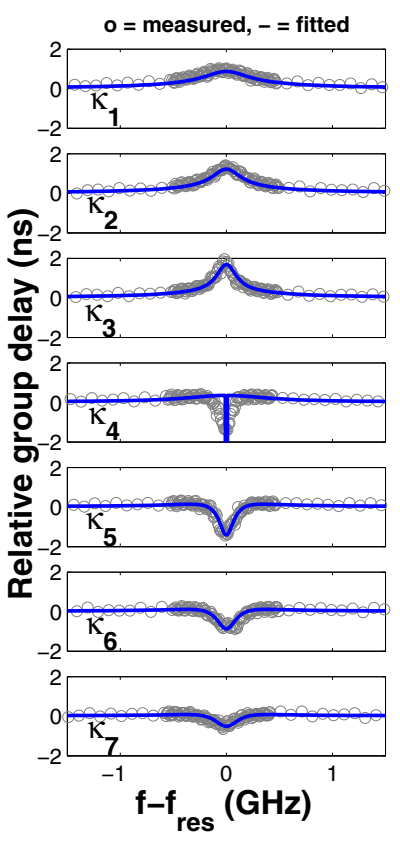

(b)
Fig. 4. (a) The measured output power (circles) and (b) the measured relative group delay (circles) and their best fit theoretical curves (solid lines).

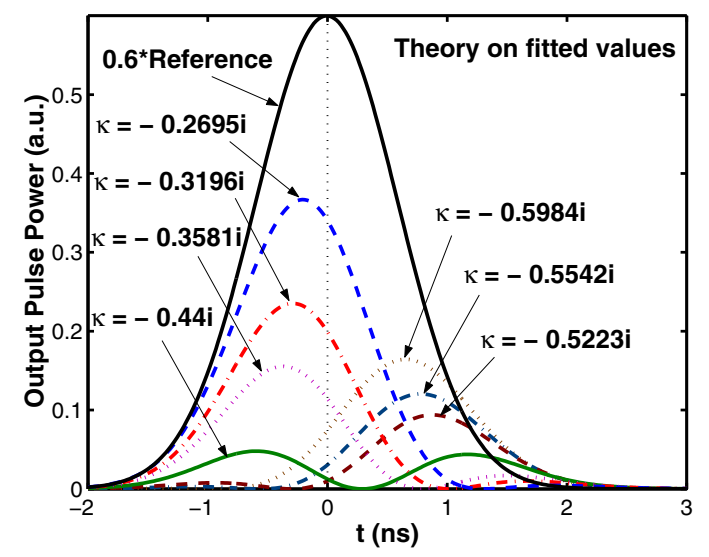

Fig. 5. The simulated output pulses using the best fit device parameter values.

Using the best fit parameter values, we applied Eqs. (8)(11) to simulate the output pulse for a Gaussian input pulse which has power profile that fits best to the normalized reference pulse. Fig. 5 shows the simulated pulse's shapes and delay which qualitatively agree to the measured pulses.
It is also interesting to note, that regardless of the fact that the group delay flips wildly when the resonator moves across the critical-coupling point, the time-domain pulse shape changes gradually from the extreme of negative delay to positive one, or vice versa, by the help of the pulse splitting phenomenon at the critical-coupling point. Note that at the critical-coupling point, the pulse does not experience an infinite delay. This comes from the fact that the pulse is not a single frequency anymore, but has spectrum which is sufficiently wide to cover also the positive part of the group delay spectrum [8].

\section{CONCLUSIONS}

We present the theory and experimental verification of pulse transmission across an optical ring-resonator circuit operating in various conditions from under-coupling, critical coupling, and over-coupling. The measured results well verify the theoretical prediction, including the negative delay phenomenon.

\section{ACKNOWLEDGMENT}

The authors thank R. G. Heideman and M. Hoekman from Lionix B.V., The Netherlands for fruitful discussions.

\section{REFERENCES}

[1] R. W. Boyd and D. J. Gauthier, "'Slow' and 'fast' light," in Prog. in Optics, Vol. 43, ed. E. Wolf, pp. 497-530. 2002.

[2] H. P. Uranus, L. Zhuang, C.G.H Roeloffzen, and H. J. W. M. Hoekstra, "Pulse Advancement and Delay in An Integrated Optical Two-Port Ring-Resonator Circuit: Direct Experimental Observations," Opt. Let., Vol. 32, No. 17, pp. 2620-2622, 2007.

[3] L. J. Wang, A. Kuzmich, and A. Dogariu, "Gain-assisted superluminal light propagation,” Nature, Vol. 406, pp. 277-279, 2000.

[4] G. M. Gehring, A. Schweinsberg, C. Barsi, N. Kostinski, R. W. Boyd, "Observation of backward pulse propagation through a medium with a negative group velocity," Science Vol. 312, pp. 895-897, 2006.

[5] M. D. Stenner, D. J. Gauthier, and M. A. Neifeld, "The speed of information in a 'fast-light' optical medium," Nature, Vol. 425, pp. 695-698, 2003.

[6] L. Brillouin, Wave propagation and group velocity, New York: Academic Press Inc., 1960.

[7] J. E. Heebner and R. W. Boyd, "Slow and fast light in resonatorcoupled waveguides," J. Modern Opt., Vol. 49, No. 14/15, pp. 26292636, 2002.

[8] H. P. Uranus and H. J. W. M. Hoekstra, "Modeling of loss-induced superluminal and negative group velocity in two-port ring-resonator circuits," J. Lightwave Technol., Vol. 25, No. 9, pp. 2376-2384, 2007.

[9] L. Zhuang, C.G.H. Roeloffzen, R.G. Heideman, A. Borreman, A. Meijerink, W. van Etten, "Single-Chip Ring Resonator-Based 1X8 Optical Beam Forming Network in CMOS-Compatible Waveguide Technology," Photon. Technol. Lett., Vol. 19, No. 15, pp. 1130-1132, 2007.

[10] R.G.Heideman, D. Geuzebroek, A. Leinse, A. Melloni, F. Morichetti, C. Roeloffzen, A. Meijerink, L. Zhuang, W. van Etten, E. Klein, and A. Driessen, "Low loss, high contrast optical waveguides based on CMOS compatible LPCVD processing," Proc. of European Conf. on Integrated Optics (ECIO) 2007, paper WB0, Copenhagen, 25-27 April 2007.

[11] http://www.lionixbv.nl.

[12] T. Niemi, M. Uusimaa, and H. Ludvigsen, "Limitations of Phase-Shift Method in Measuring Dense Group Delay Ripple of Fiber Bragg Gratings," Photon. Technol. Lett., Vol. 13, No. 12, pp. 1334-1336, 2001.

[13] M. Mojahedi, K. J. Malloy, G. V. Eleftheriades, J. Woodley, and R. Y. Chiao, "Abnormal wave propagation in passive media," J. Sel. Topics in Quantum Elect., Vol. 19, No. 1, pp. 30-39, 2003. 